\title{
Marker-free cell discrimination by holographic optical tweezers
}

F. Schaal

schaal@ito.uni-stuttgart.de

\section{Warber}

\section{S. Zwick}

\section{H. van der Kuip}

T. Haist

W. Osten
University of Stuttgart, Institut für Technische Optik, Stuttgart, 70569, Germany

University of Stuttgart, Institut für Technische Optik, Stuttgart, 70569, Germany.

University of Stuttgart, Institut für Technische Optik, Stuttgart, 70569, Germany.

Dr. Margarete Fischer-Bosch Institute of Clinical Pharmacology and University of Tuebingen, 70376, Germany.

University of Stuttgart, Institut für Technische Optik, Stuttgart, 70569, Germany.

We introduce a method for marker-free cell discrimination based on optical tweezers. Cancerous, non-cancerous, and drug-treated cells could be distinguished by measuring the trapping forces using holographic optical tweezers. We present trapping force measurements on different cell lines: normal pre-B lymphocyte cells (BaF3; "normal cells"), their Bcr-Abl transformed counterparts (BaF3-p185; "cancer cells") as a model for chronic myeloid leukaemia (CML) and Imatinib treated BaF3-p185 cells. The results are compared with reference measurements obtained by a commercial flow cytometry system. [DOI: 10.2971/jeos.2009.09028]

Keywords: optical tweezers, marker free cell discrimination, chronic myeloid leukaemia

\section{INTRODUCTION}

Discrimination of different cells and cell states is important for identification of diseases, treatment monitoring, and indication of drug response in clinical diagnosis and biomedical research. Therefore, a lot of methods have been developed in the past for differentiating cells. Due to their high speed and the contactless operation, optical methods are especially useful for this purpose.

Image-processing based techniques that classify different cells have already attained impressive results, but for many applications methods which purely rely on simple dyeing or phasecontrast techniques are not powerful enough. Figure 1 shows a typical example that will be used later on in this article: the cancerous and non-cancerous white blood cells look exactly the same. Discrimination based solely on images is not possible. Therefore, often more sophisticated methods are necessary.
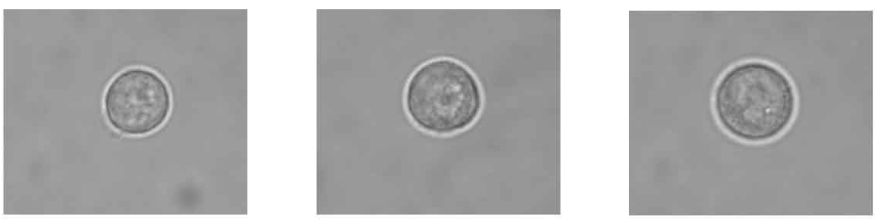

FIG. 1 Microscopic bright-field images of BaF3 ("normal cells"), BaF3-p185 ("cancer cells") and Imatinib treated BaF3-p185 cells
Most often, the binding of specially marked molecules to their corresponding targets is employed. Detection is then performed manually or in an automated way based on imaging or flow cytometry. Fluorescence-based methods (e.g. fluorescence activated cell sorting (FACS)) are especially widely used in immunology [1,2]. Applications for such techniques range from the simple diagnosis of pregnancy to the detection of cancerous cells.

Unfortunately, the application of marker-based methods also has some drawbacks. Most importantly, a suitable marker first has to be developed. Such a development (including approval) is typically very expensive. Additionally, often laborious biochemical preparation has to be performed before marking is possible. This potentially leads to influences on the cells and the measurement results, e.g. by reducing the cell viability. Due to these disadvantages, a lot of research is currently directed towards the development of marker-free detection methods.

Today, optical marker-free detection in a clinical environment is especially based on stray-light analysis [3]-[5] (e.g. using Doppler anemometers or interference-based setups) but a lot of other techniques can also deliver important information. Examples are autofluorescence-based methods, spectroscopy [6] (e.g. Raman-based), and quantitative phase contrast microscopy $[7,8]$. The incorporation of the behaviour over time 
(e.g. time-gated fluorescence) might also give valuable information [9].

A new class of optical methods for diagnosis employs the action of force that light is able to exert onto relevant particles like cells or viruses. Specifically, it is possible to measure the mechanical parameters of different cell states by systematically deforming the cells using optical cell stretchers [10][12]. Another interesting class of methods measures the forcecoupling to secondary objects (typically coated or uncoated microbeads) [13]. These methods are very powerful for clinical research but not very much suited for a cost-effective automated system.

Ladavac et al. proposed a system where cells are sorted based on their movement in a light field. [14] Such "optical sorting" methods have shown themselves to be sensitive of the refractive index and the size of the cell [15]. Additionally, Dharmadhikari et al. and Mohanty et al. have shown that opticalinduced rotation of erythrocytes can be used for the early detection of malaria [16, 17]. The classification of HeLa cells [18], viruses [3], and yeast cells [19] has also been demonstrated. Martin et al. [12] have shown the discrimination of different breast cancer cells by measuring the optical deformabiliy.

In the following we describe a method that uses the lightinduced force for discriminating cells that are indistinguishable by conventional image analysis (Figure 1). Optical tweezers are able to trap and move micro-objects. The trapping force depends on the shape, the structure, and the relative index of refraction of the object and the surrounding medium. Different cells or cells in a different state lead to different trapping forces, depending on the cell type, cell structure, metabolic activity, and drug treatment.

We employ a holographic optical tweezers (HOT) system for performing escape-force measurements on pre-B lymphocyte cells. Section 2 describes the optical setup and in Section 3 we show the results for these cells as well as reference measurements obtained with a commercial flow cytometry system. Finally, we give a short summary and an outlook in Section 4.

\section{EXPERIMENTAL SETUP}

The method uses a system based on holographic optical tweezers [20]. The setup (Figure 2) consists of a $20 \mathrm{~W}$ diode pumped Nd:YAG laser $(\lambda=1064 \mathrm{~nm})$ running at $4,9 \mathrm{~W}$, which illuminates a reflective phase-only spatial light modulator (Holoeye HEO 1080P) with 2 million pixels, $8 \mu \mathrm{m}$ pixel pitch and $2 \pi$ phase modulation at $\lambda=1064 \mathrm{~nm}$. A phase hologram is computed and written into the light modulator. Optical traps are then generated by reconstructing the hologram in the object plane of the microscope. By the digital hologram we are able to generate hundreds of optical traps and move them independently in all three dimensions with a positioning accuracy better than $10 \mathrm{~nm}$.

To allow real time movement of the traps, the hologram computation is done on a graphics board (Nvidia 6800 GT) [21]. The system is operating at a wavelength of $\lambda=1064 \mathrm{~nm}$ to minimize the damage by light absorption in biological objects [22]. The light modulator and the microscope (Zeiss Axiovert 200M) are coupled by a telescope and a dichroic beam splitter. A detailed analysis of the setup is described by Haist et al. [23]. The high numerical aperture water immersion lens (Zeiss C-Apochromat $40 \times$ NA 1.2) is used to obtain good trapping efficiency and high resolution imaging. Images of the object plane were recorded using a Zeiss AxioCam MRm CCD camera.

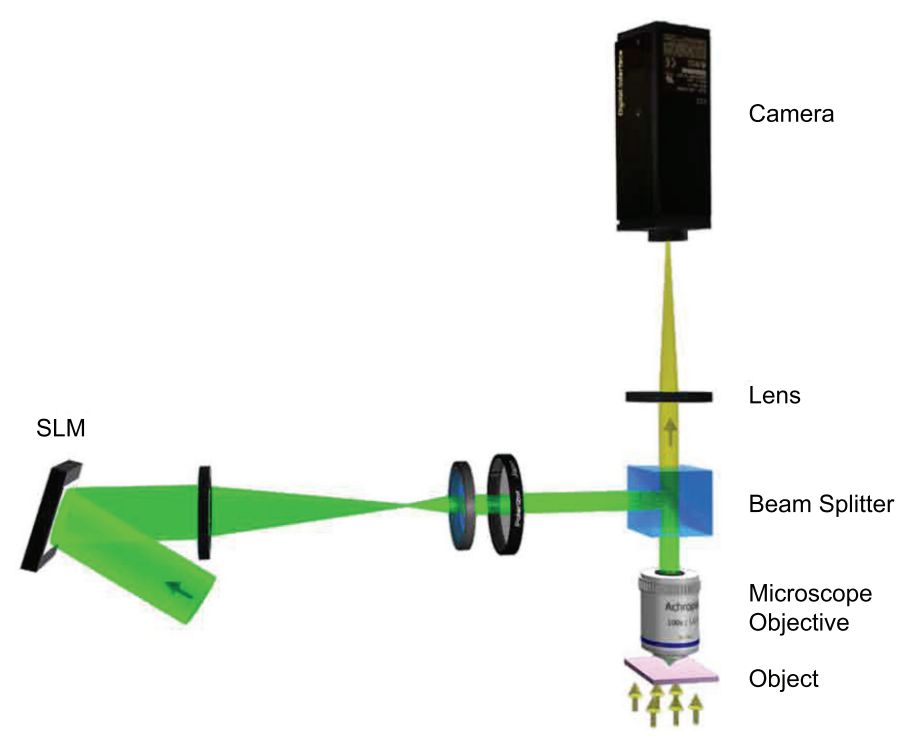

FIG. 2 Setup of the holographic optical tweezers

Several methods for measuring the trapping force have been proposed in the past. Examples are techniques based on Brownian motion [24], oscillation [25], deflection or escape force [26]. To keep the measurement time short we did not consider methods based on thermal fluctuations. Therefore we have chosen a trapping force measurement method, based on Stokes friction (escape force method). The cells are trapped at the holographically generated foci and accelerated until they leave the trap due to the viscous drag. The geometry and maximum speed of the cells are measured by image processing. The trapping forces are calculated using Stokes law. Measurements were done on single cells with one optical trap. The cells were trapped manually and lifted to a height of $50 \mu \mathrm{m}$ above the cover glass surface. To avoid interferences with other cells, we used a cell concentration of 100.000 cells $/ \mathrm{ml}$ and discarded measurements with more than one cell inside the microscope field.

We developed an image processing algorithm (Figure 3) to track the cell movement and measure the cell diameter. To detect the cells the edges were processed by a Canny algorithm [27]. The output of the Canny algorithm is a binary image. Possible small gaps on the edges are closed by dilatation. After closing, the original cell diameter is restored by erosion. Small areas are deleted in order to remove all filled areas beside the cell, e.g. other particles and artefacts. After that, the area of the cell is filled. The cell position is computed as the centroid of the filled area and the cell diameter is computed by a circle fit of the cell profile. The cell diameter of moving cells measured with this method has an average standard deviation of $0,2 \mu \mathrm{m}$. 

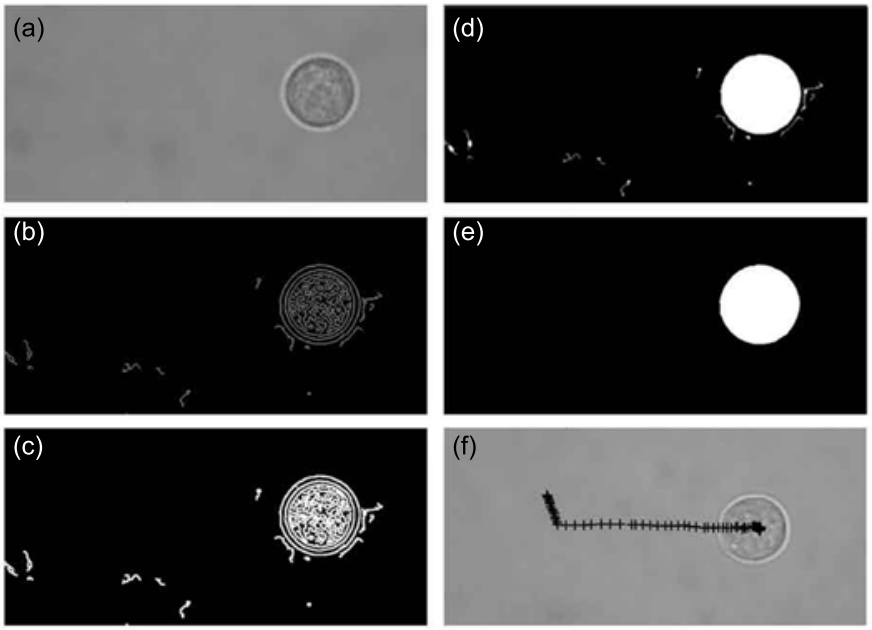

FIG. 3 Image processing: (a) camera image, (b) image after Canny algorithm, (c) image after dilatation, (d) filling of closed areas and erosion, (e) removal of small areas, ( $f$ ) cell position during a measurement (100 images).

The cell speed is determined by differentiation of the time dependent cell position (Figure 4). Internal flows in the surrounding medium dragging the cells, might lead to wrong measurements results. This is corrected by measuring the speed of the cell after falling out of the trap and subtracting this base speed from the maximum speed. Trapping force calculation based on Stokes law for spherical particles is valid due to the laminar flow around the cells $(d \approx 12 \mu \mathrm{m})$ and the spherical shape of the measured cells. The trapping force is then given by

$$
F=6 \pi r \eta v_{\max }
$$

where the cell radius is denoted by $r$. The maximum cell speed is $v_{\max }$ and the dynamic viscosity of the used medium RPMI 1640 (see Section 3) $\eta$ equals $8.4 \times 10^{-4} \mathrm{Ns} / \mathrm{m}^{2}$.

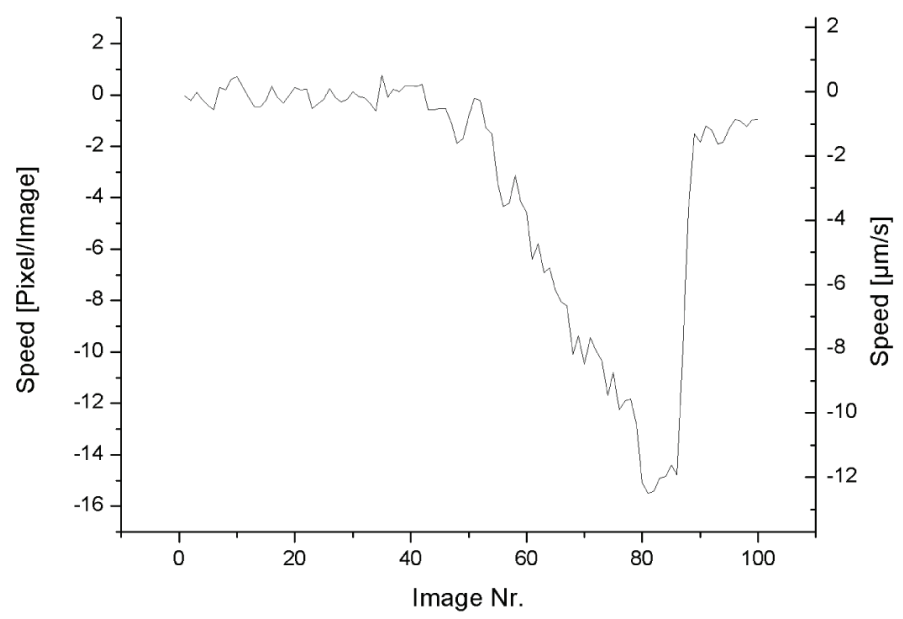

FIG. 4 Cell speed during a trapping force measurement of a single cell. The cell is trapped in a static optical trap between image 0 and 40 . The cell is only moving due to the Brownian motion. The optical trap is moving with increasing speed from image 40. The cell leaves the optical trap, due to the increasing flow resistance at a maximum speed of $-12.4 \mu \mathrm{m} / \mathrm{s}$ at image 81 . The cell quickly slows down and is dragged by the surrounding flow in the medium with an average speed of $-1,1 \mu \mathrm{m} / \mathrm{s}$, between image 84 and 100.

\section{EXPERIMENTAL RESULTS}

Measurements were taken on different cell lines: normal preB lymphocyte cells (BaF3; "normal cells"), their Bcr-Abl transformed counterparts (BaF3-p185; "cancer cells") as a model for chronic myeloid leukemia (CML) and imatinib treated BaF3-p185 cells. Imatinib is a molecularly targeted drug for CML cancer treatment [28].

The parental murine pre-B lymphocyte cell line BaF3 and the p185 Bcr-Abl expressing derivative thereof, BaF3p185, were a kind gift from Prof. J. Duyster (Munich, Germany). BaF3p185 was transformed with pSLXBcr-Abl. Parental BaF3 cells were grown in RPMI 1640 (Biochrom, Berlin, Germany) complemented with $10 \%$ fetal calf serum, glutamine, and $1 \mathrm{ng} / \mathrm{mL}$ recombinant murine IL-3 (PBH, Hannover, Germany) whereas the factor independent BaF3-p185 cells were grown without additional growth factor supplementation.

Imatinib mesylate (STI571; Glivec) is a specific inhibitor of the $\mathrm{c}-\mathrm{Abl}$ and Bcr-Abl-kinase and was purchased from Toronto Research Chemicals inc. (North York, Canada). A stock solution $(10 \mathrm{mg} / \mathrm{ml})$ was prepared by dissolving the compound in DMSO $/ \mathrm{H}_{2} \mathrm{O}(1: 1)$ and kept at $-20^{\circ} \mathrm{C}$. Cells were incubated with or without $3 \mu \mathrm{M} / \mathrm{ml}$ Imatinib for $120 \mathrm{~min}$. and then harvested.

Trapping force measurements for a series of 200 cells were taken of BaF3, BaF3-p185 and 2 hours Imatinib treated BaF3p185 cells. Each measurement series was repeated once. The trapping force distribution of BaF3 and BaF3-p185 cells is shown in Figure 5. Figure 6 contains the trapping force distribution of BaF3-p185 and Imatinib treated cells. A Gaussian curve is fitted on histograms of the trapping force distributions. The histograms of cancerous and normal cells (Figure 5) can be clearly distinguished by their standard deviation. The standard deviation of the cancerous cells $\left(3.5 \times 10^{-13} \mathrm{~N}\right)$ is significantly wider than the standard deviation of normal cells $\left(1.8 \times 10^{-13} \mathrm{~N}\right)$. Cancerous and drug-treated cancerous cells (Figure 6) can be separated by their peak values. The trapping force distribution peak value of cancerous cells $\left(1.2 \times 10^{-12} \mathrm{~N}\right)$ is smaller than the peak value of Imatinib treated cancer cells $\left(1.4 \times 10^{-12} \mathrm{~N}\right)$.

We also performed flow cytometry measurements of BaF3p185 and $3 \mu \mathrm{M} / \mathrm{ml}$ Imatinib treated BaF3-p185 cells after different incubation times (Figure 7). The fluorescent markers for early cell death processes TMRM and Annexin V were used to distinguish untreated and Imatinib treated BaF3-p185 cells. In contrast to the trapping force based method untreated and Imatinib treated cells could not be distinguished after 2 hours incubation.

Moehring et al. [29] measured a rapid increase of poly(ADPribosyl)ation in BaF3-p185 cells after 2 hours Imatinib exposure by Western blot analysis. This indicates differences in the molecular structure between Imatinib treated and untreated BaF3-p185 cells after 2 hours Imatinib exposure.

We measured no significant discrepancy between the cell diameter distributions (Figure 8) of the different cell types. Also 


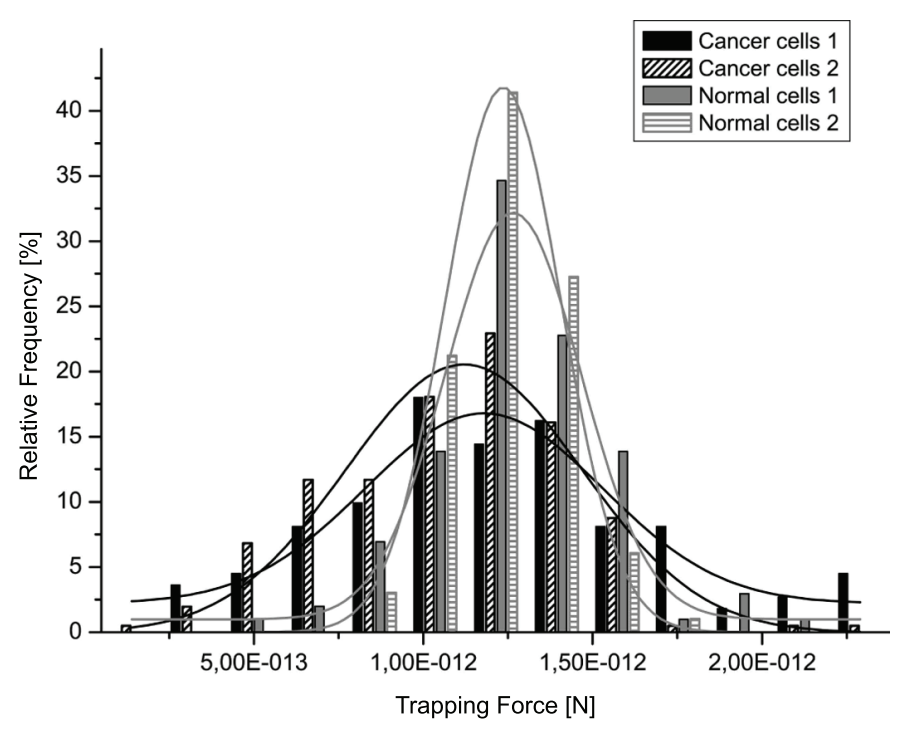

FIG. 5 Trapping forces of BaF 3 cells ("normal cells") and BaF3-p185 cells ("cancer cells")

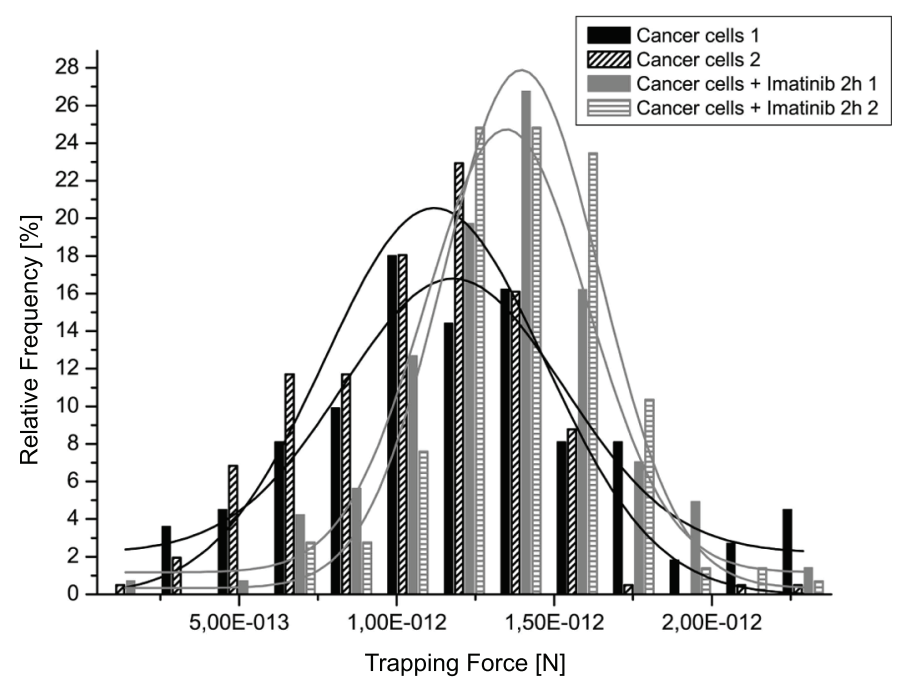

FIG. 6 Trapping forces of BaF3-p185 cells ("cancer cells") and Imatinib treated BaF3p185 cells after 2 hours drug exposure

no significant change in the cell cross sectional area during a trapping force measurement was observed.

The cause for the observed effect is not identified yet. Somehow the interaction of light with the cell is changed by the different cell state. This might be due to a simple change of refractive index or shape, but might be also due to much more complicated issues. Absorption and light deflection due to the internal cell structures is in a complicated way also important for the transfer of momentum and therefore force. This means that one would have to know this detailed fine structure and its changes and then one would have to model the light deflection using some sort of rigorous electrodynamic modelling. We think that this is not possible at the state of art. Still, medical application is possible using the proposed method/results.

\section{SUMMARY AND OUTLOOK}

We have shown that holographic optical tweezers can be used to discriminate between normal, cancerous and drug-

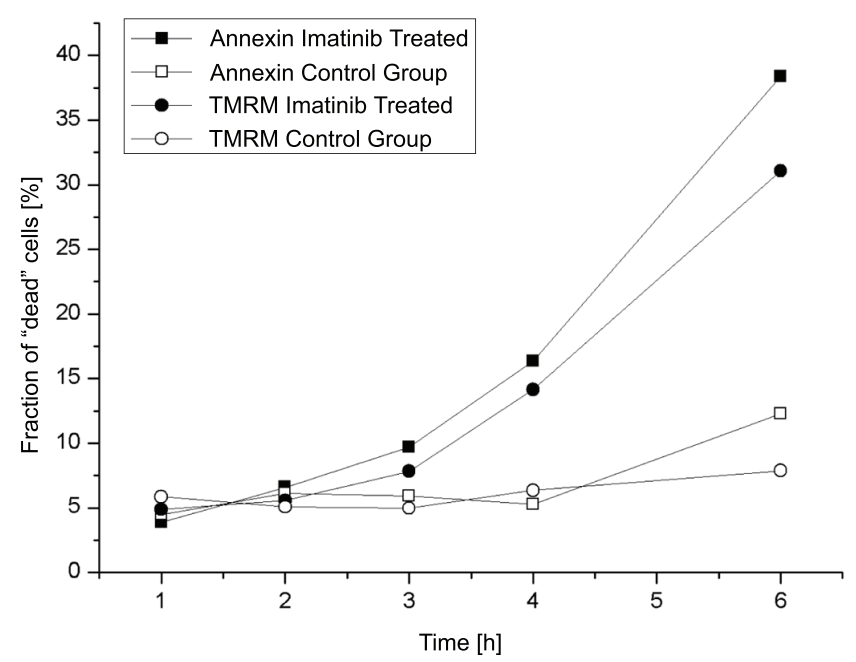

FIG. 7 FACS measurements of Imatinib treated and untreated BaF3-p185 cells ("cancer cells") marked with the fluorescent markers for early cell death processes TMRM and Annexin $\mathrm{V}$

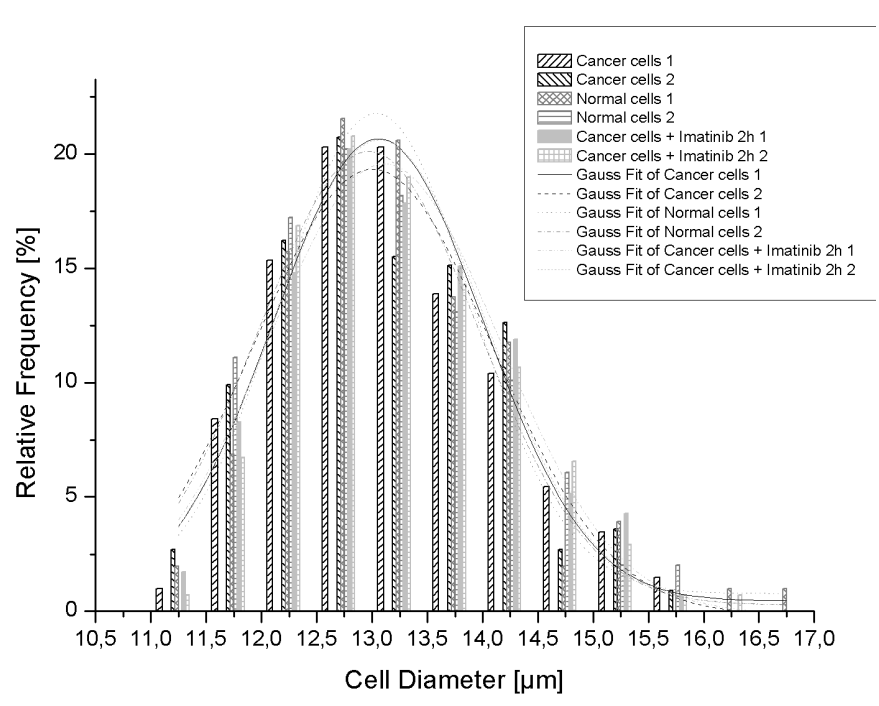

FIG. 8 Cell diameter distribution

treated cancerous leucocytes without using additional markers. Moreover, the optical force that a focused light beam exerts onto the cell seems to be more sensitive to changes of the cell than the standard FACS-based measurement with fluorescence-based markers. For the force measurement we use an escape force approach using image processing-based evaluation. In principle, the method can be completely automated so that diagnosis without additional markers should be possible in a cost effective way for isolated cells.

Compared to most other methods there is no need for difficult or time-consuming preparation of the cells and the cells are not damaged by the measurement. Sorting of the discriminated cells by active movement using the holographic technique is also in principle possible. Since only one integral parameter, namely the force acting onto the particle, is measured it might be advantageous to combine the method with other techniques that deliver additional information. We think that the combination with phase contrast imaging and morphology-based features is especially interesting for a broad range of applications. 


\section{ACKNOWLEDGEMENT}

We thank the Bundesministerium für Bildung und Forschung for financial support within the project "AZTEK" (FKZ 13N8809).

\section{References}

[1] G. Sluder and D. Wolf, "Digital Microscopy" Method. Cell Biol. 72, (2003).

[2] K. Koenig, "Multiphoton microscopy in life sciences" J. Microsc.0xford 200, 83-104 (2000).

[3] F. Ignatovich, D. Tophan, and L. Novotny, "Optical Detection of Single Nanoparticles and Viruses" IEEE J. Sel. Top. Quant. 12, 12921300 (2006).

[4] R. Doornbos, M. Schaeffer, A. Hoekstra, P. Sloot, B. De Grooth, and J. Greve, "Elastic light-scattering measurement of single biological cells in an optical trap" Appl. Optics 35, 729-734 (1996).

[5] P. Wyatt, "Identifcation of bacteria by differential light scattering" Nature 221, 1257-1258 (1969).

[6] P. Roesch, M. Schmitt, W. Kiefer, and J. Popp, "The identification of microorganisms by micro-raman spectroscopy" J. Mol. Struct. 661-662, 363-369 (2003).

[7] B. Kemper, D. Carl, and G. von Bally, "Digitalholographische Mikroskopie: Ein Verfahren zur markerfreien dynamischen Analyse lebender Zellen" Photonik 2, 44-47 (2006).

[8] B. Kemper and G. von Bally, "Digital holographic microscopy for live cell applications and technical inspection" Appl. Optics 47, 52-61 (2008).

[9] H. Schneckenburger, R. Sailer, A. Hendinger, M. Bauer, and L. Strauss, "Phagotosis: Studies by optical tweezers and timeresolved microspectrofluorometry" Proc. SPIE 3568, 148-153 (1998).

[10] J. Guck, R. Ananthakrishnan, T. Moon, C. Cunningham, and J. Kaes, "Optical Deformability of Soft Biological Dielectrics" Phys. Rev. Lett. 84, 5451-5454 (2000).

[11] J. Guck, R. Ananthakrishnan, H. Mahmood, T. Moon, C. Cunningham, and J. Kaes, "The optical stretcher: A novel laser tool to micromanipulate Cells" Biophys. J. 81, 767-784 (2001).

[12] M. Martin, K. Mueller, F. Wottawah, S. Schinkinger, B. Lincoln, M. Romeyke, and J. Kaes, "Feeling with Light for Cancer" Proc. SPIE 6080, 60800P-1 (2006).

[13] P. Seitz, E. Stelzer, and A. Rohrbach, "Interferometric tracking of optically trapped probes behind structured surfaces: a phase correction method" Appl. Optics 45, 7309-7315 (2006).

[14] K. Ladavac and D. Grier, "Sorting mesoscopic objects with periodic potential landscapes: optical fractionation" Phys. Rev. E 70, 10901 (2004).
[15] M. MacDonald, G. Spalding, and K. Dholakia, "Microfluidic sorting in an optical lattice" Nature 426, 421-424 (2003).

[16] J. Dharmadhikari, S. Roy, J. Dharmadhikari, S. Sharma, and D. Mathur, "Torquegenerating malaria-infected red blood cells in an optical trap" Opt. Express 12, 1179 (2004).

[17] S. Mohanty, A. Uppal, and P. Gupta, "Self-rotation of red blood cells in optical tweezers: prospects for high throughput malaria diagnosis" Biotechnol. Lett. 26, 971-974 (2004).

[18] S. Hoo, M. Wang, J. Kohrumel, and J. Hall, "A Novel Method for Detection of Virus-Infected Cells Through Moving Optical Gradient Fields Using Adenovirus as a Model System" Cytom. Part A 48A, 140-146 (2004).

[19] M. Wang, C. Schnabel, M. Chachisvilis, R. Yang, M. Paliotti, L. Simons, L. McMullin, N. Hagen, K. Lykstad, E. Tu, L. Pestana, S. Sur, H. Zhang, W. Butler, I. Kariv, and P. Marchand "Optical forces for non-invasive cellular analysis" Appl. Optics 42, 5765-5773 (2003).

[20] M. Reicherter, T. Haist, E. Wagemann, and H. Tiziani, "Optical particle trapping with computer-generated holograms written on a liquid-crystal display" Opt. Lett. 24, 608-610 (1999).

[21] M. Reicherter, T. Haist, S. Zwick, A. Burla, L. Seifert, and W. Osten, "Fast hologram computation and aberration control for holographic tweezers" Proc. SPIE 5930, 501-509 (2005).

[22] K. Svoboda and S. Block, "Biological Applications of Optical Forces" Annu. Rev. Biophys Biom. 23, 247-285 (1994).

[23] T. Haist, S. Zwick, M. Warber, and W. Osten, "Spatial Light Modulators - Versatile Tools for holography" J. Holography Speckle 3, 1-12 (2006).

[24] E. Florin, A. Pralle, E. Stelzer, and J. Hoerber, "Photonic force microscope calibration by thermal noise analysis" Appl. Phys. A-Mater. 66, (1998).

[25] M. Andersson, A. Madgavkar, M. Stjerndahl, Y. Wu, S. Niehren, K. Mustafa, K. Arvidson, W. Wennerberg, A. Tan, and R. Duran, "Using optical tweezers for measuring the interaction forces between human bone cells and implant surfaces: System design and force calibration" Rev. Sci. Instrum. 78, 074302 (2007).

[26] R. Simmons, J. Finer, S. Chu, and J. Spudich, "Quantitative Measurements of Force and Displacement Using an Optical Trap" Biophys. J. 70, 1813-1822 (1996).

[27] R. Gonzalez and R. Woods, Digital Image Processing, 3 edn. (Prentice Hall International, 2003).

[28] M. Henkes, H. van der Kuip, and W. Aulitzky, "Therapeutic options for chronic myeloid leukemia: focus on imatinib" Ther. Clin. Risk. Manag. 4, 1-26 (2008).

[29] A. Moehring, L. Wohlbold, W. Aulitzky, and H. van der Kuip, "Role of poly(ADP-ribose) polymerase activity in imatinib mesylateinduced cell death" Cell Death Diff. 12, 627-636 (2005). 